\title{
From period-doubling to folding in stiff film/soft substrate system: The role of substrate nonlinearity
}

\author{
Lijun Zhuo ${ }^{1}$, Yin Zhang * \\ State Key Laboratory of Nonlinear Mechanics, Institute of Mechanics, Chinese Academy of Sciences, Beijing 100190, China
}

\section{A R T I C L E I N F O}

\section{Article history:}

Received 29 January 2015

Received in revised form

1 May 2015

Accepted 4 May 2015

Available online 12 May 2015

Keywords:

Period-doubling

Folding

neo-Hookean

Substrate nonlinearity

Pre-stretch

\begin{abstract}
A B S T R A C T
Uniaxial compressed stiff films on soft substrates can evolve into the period-doubling and folding instabilities, beyond the onset of sinusoidal wrinkling. The substrate is modeled as a neo-Hookean solid with a pre-stretch prior to film attachment, and its nonlinearity is obtained. Both the pre-stretch and the external nominal strain imposed on the film/substrate system can induce different substrate nonlinearity, and thus have different effects on the post-buckling mode evolution of the system. This study shows that the critical strain of period-doubling instability is linear to the pre-stretch. As the compressive nominal strain increases, the folding mode occurs beyond the onset of period-doubling in both the pre-tension and the pre-compression case, due to the softening/hardening effects for the inward/outward displacements generated by the positive substrate nonlinearity.
\end{abstract}

(c) 2015 Elsevier Ltd. All rights reserved.

\section{Introduction}

Thin stiff films bound to thick compliant substrates can lose stability when the in-plane compressive stress exceeds a critical value, giving rise to micro and nano-scale wrinkles [1]. Such instability phenomenon is apparent in nature, such as human skins [2], tubular organs of animals [3] and hard exocarp/soft sarcocarp plants $[4,5]$. The buckling of the stiff film/compliant substrate system has been treated as a desirable means of generating various self-organized patterns with intrinsic wavelengths [6-8]. It finds a broad range of potential applications in, for instance, the thin-film metrology [9,10], tunable optical gratings [11,12], and stretchable electronics which can undergo a considerable deformation with the internal stress being less than the material strength [13-16].

The post-buckling behavior of film/substrate systems has received increasing attention due to the variety of instability modes. The primary instability modes include wrinkling [17], creasing [18,19], and buckle delamination [20,21], while the secondary bifurcation modes consist of period-doubling [22], period-tripling [23], localized ridges [24,25] and folds [26,27]. If a film is well-bonded to a substrate, and the film modulus is much higher than that of the substrate, the buckle delamination and creasing instability can be avoided $[25,28]$. Therefore, the initial flat state of the thin film will lose stability and switch into

\footnotetext{
* Corresponding author. Tel.: +8610 82543970; fax: +86 1082543935 .

E-mail address: zhangyin@Inm.imech.ac.cn (Y. Zhang).

${ }^{1}$ Present address: Center for Composite Materials, Harbin Institute of Technology, Harbin 150080, China.
}

sinusoidal wrinkles when the compressive stress reaches the onset of wrinkling. The compression is commonly generated by the release of substrate pre-stretch imposed prior to film attachment $[7,8,14]$. Further compression of the system can induce perioddoubling [22], period-tripling [23], folds [27] and localized ridges [25]. Some previous studies revealed that the substrate pre-stretch and the film/substrate modulus ratio determine the occurrence and evolution of the post-buckling modes [23-25,28]. Localized ridges appear when the pre-stretch is sufficiently large and folds form in systems that have low film/substrate modulus ratio $[25,28]$. The period-doubling configuration forms in a wide range of pre-stretch and modulus ratios [25,28]. The period-tripling morphology is identified in the low modulus ratio case, and can occur instead of period-doubling at a later secondary bifurcation point [23]. However, the quantitative connections between the onset of these post-buckling modes and the system parameters have not been established. In this study, we focus on the perioddoubling instability, and an approximate relationship governing the onset of this instability mode is presented.

Some theoretical and numerical investigations have focused on the role of substrate nonlinearity in the occurrence of the various post-buckling modes [22,24,29-31]. An elastic strut supported by an elastic foundation experiences mode jumping due to the stiffening effect of the cubic nonlinearity [31,32]. Zhuo and Zhang [33] revealed that the substrate quadratic nonlinearity in the stiff film/soft substrate system causes mode coupling and then induces the period-doubling, which is regarded as a pitchfork bifurcation [34]. The experiment carried by Brau et al. [22] also showed that the period-doubling instability is triggered by the substrate quadratic nonlinearity, which induces asymmetric traction-displacement 
relation: the normal tension/compression on the substrate surface is no longer equivalent for the same given outward/inward displacement. In addition, the pre-tension and pre-compression imposed on the substrate prior to film attachment have different effect on the substrate nonlinearity, giving rise to the formation of localized ridges and folds, respectively [24,25]. Hutchinson [30] established the relation between the pre-stretch and the nonlinearity of a neo-Hookean substrate and identified that the pre-stretch is one of the parameters that control the instability of the film/substrate bilayer. In this study, the expression for the nonlinear effect is further given as a function of the pre-stretch and the overall nominal strain. Moreover, the mode transition from period-doubling to folding is possible as the compression increases considerably.

This study explores the role of substrate nonlinearity in the morphology evolution of a compressed stiff film on a compliant substrate. Based on the perturbation method, the nonlinear response of a neo-Hookean half-space under periodic displacement constraints is presented and the substrate nonlinearity is obtained. Finite element simulations of neo-Hookean film/substrate systems are also carried out, which show the morphology transitions from sinusoidal wrinkling to period-doubling and then the folding mode. The model also predicts the critical strain of period-doubling instability.

\section{Nonlinear traction-displacement behavior of a neo- Hookean substrate}

In this section, a perturbation analysis is presented to analyze the nonlinear response of a semi-infinite neo-Hookean substrate which undergoes plane-strain deformation. Fig. 1 illustrates three different states during the wrinkling of a stiff thin film/soft substrate bilayer. An elastomeric (e.g., poly(dimethylsiloxane)
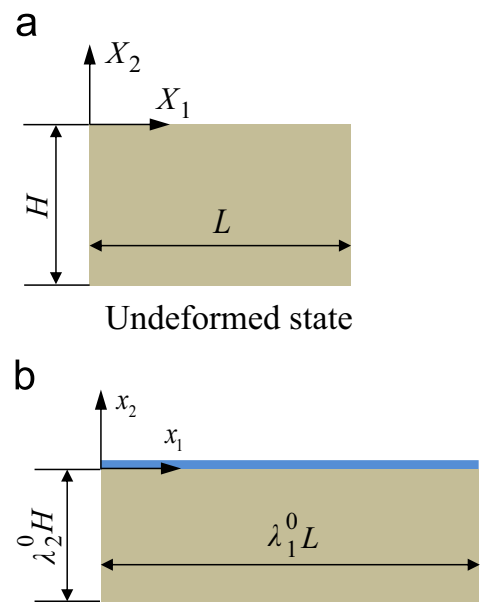

Prestretched state

C

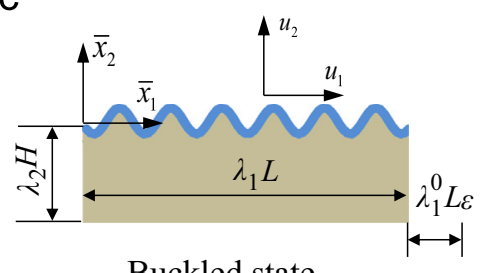

Buckled state

Fig. 1. Schematics of the wrinkling process of the film/substrate bilayer and the coordinate systems. (a) The initially undeformed substrate of length $L$ and of height $H$. (b) The substrate undergoes a pre-stretch $\lambda_{1}^{0}$ before attaching the thin film. (c) The film is buckled and the substrate stretch is $\lambda_{1}$ compared to the undeformed state. The nominal compressive strain applied on the bilayer is $\varepsilon=\left(\lambda_{1}^{0}-\lambda_{1}\right) / \lambda_{1}^{0}$.
(PDMS)) substrate of length $L$ and height $H$ in the undeformed state (Fig. 1(a)) is uniformly pre-stretched prior to the attachment of a stiff thin film (Fig. 1(b)). Lagrangian coordinates, $X_{i}$ and $x_{i}$ $(i=1,2)$, specify the material points in the undeformed state and pre-stretched state, respectively. The pre-stretches in three principal axes $X_{1}, X_{2}$ and $X_{3}$ are denoted by $\lambda_{1}^{0}, \lambda_{2}^{0}$ and $\lambda_{3}^{0}$, respectively. To characterize the plane-strain deformation, the pre-stretch in $X_{3}$ direction is set zero, i.e., $\lambda_{3}^{0}=1$. The material is assumed to be incompressible, i.e., $\lambda_{1}^{0} \lambda_{2}^{0}=1$. The coordinates of the material points with respect to the pre-stretched state are related to that of the undeformed state by the following

$x_{i}=\lambda_{i}^{0} X_{i} \quad(i=1,2)$

In the buckled state (Fig. 1(c)), the coordinates are denoted by

$\left\{\begin{array}{l}\bar{x}_{1}=\lambda_{1} X_{1}+u_{1} \\ \bar{x}_{2}=\lambda_{2} X_{2}+u_{2}\end{array}\right.$,

where $u_{1}$ and $u_{2}$ are the displacements parallel to the coordinate axes $X_{1}$ and $X_{2}$, respectively. $\lambda_{i} \quad(i=1,2)$ is the stretch of the substrate, which is defined as the length in the current state (the buckled state) divided by the length in the reference state (the undeformed state). It is related to the pre-stretch by

$\left\{\begin{array}{l}\lambda_{1}=\lambda_{1}^{0}(1-\varepsilon) \\ \lambda_{2}=\lambda_{2}^{0} /(1-\varepsilon)\end{array}\right.$

where $\varepsilon=\left(\lambda_{1}^{0}-\lambda_{1}\right) / \lambda_{1}^{0}$ is the nominal strain (positive for compression), as shown in Fig. 1(c). The strain $\varepsilon$ should be compressive to induce the buckling of the film/substrate system, which means that the stretch $\lambda_{1}<\lambda_{1}^{0}$ from Eq. (3).

The substrate is assumed to be an incompressible neo-Hookean material and hence its strain energy densities in the pre-stretched $\left(W_{\mathrm{I}}\right)$ state and the buckled state $\left(W_{\mathrm{II}}\right)$ are given as follows [35]

$W_{\mathrm{I}}=\frac{\mu_{s}}{2}\left[\left(\lambda_{1}^{0}\right)^{2}+\left(\lambda_{2}^{0}\right)^{2}-2\right]$

and

$W_{\mathrm{II}}=\frac{\mu_{s}}{2}\left[\left(\lambda_{1}+u_{1,1}\right)^{2}+u_{2,1}^{2}+\left(\lambda_{2}+u_{2,2}\right)^{2}+u_{1,2}^{2}-2\right]$.

Therefore, the increment of the strain energy density in passing from the pre-stretched state to the buckled state is thus given as the following

$\Delta W=W_{\text {II }}-W_{\mathrm{I}}=\mu_{s}\left[\frac{1}{2}\left(u_{1,1}^{2}+u_{1,2}^{2}+u_{2,1}^{2}+u_{2,2}^{2}\right)+\lambda_{1} u_{1,1}+\lambda_{2} u_{2,2}+f_{\varepsilon}\right]$,

where $f_{\varepsilon}=\left(\lambda_{1}^{0}\right)^{2}\left[(1-\varepsilon)^{2}-1\right]+\left(\lambda_{2}^{0}\right)^{2}\left[1 /(1-\varepsilon)^{2}-1\right] ; \quad \mu_{s}=E_{s} /$ $\left[2\left(1+\nu_{s}\right)\right]$ is the shear modulus of the substrate; $E_{s}$ and $\nu_{s}$ are the Young's modulus and Poisson's ratio, respectively.

The incompressibility condition of the substrate in the buckled state is written as [35]

$J-\lambda_{1} \lambda_{2}=\lambda_{2} u_{1,1}+\lambda_{1} u_{2,2}+u_{1,1} u_{2,2}-u_{1,2} u_{2,1}=0$,

where $J$ is the determinant of the deformation gradient, namely, the volume change from the reference state to the current state.

Let $Q=Q\left(X_{1}, X_{2}\right)$ be the function to be determined which has the same period as the surface displacements [19,30]. Here a Lagrangian multiplier $q$ is introduced to enforce the incompressibility condition in Eq. (7) [30]. Physically, the Lagrangian multiplier $q$ indicates the hydrostatic pressure, namely, $q=q_{0}+\Delta q=$ $\mu_{s}(r+Q)$, where $r=\lambda_{2} / \lambda_{1}=1 / \lambda_{1}^{2} . q_{0}=\mu_{s} r$ is the pressure in the pre-stretched state and $\Delta q=\mu_{s} Q$ is the increment of the pressure from the pre-stretched state to the buckled state [26]. The change of the total potential energy in passing from the pre-stretched 
state to the buckled state is therefore $[19,30]$

$$
\begin{aligned}
\phi & =\int_{V}\left[\Delta W-q\left(J-\lambda_{1} \lambda_{2}\right)\right] d V-\phi_{s} \\
= & \mu_{s} \int_{0}^{l_{0}} \int_{-\infty}^{0}\left[\frac{1}{2}\left(u_{1,1}^{2}+u_{2,2}^{2}+u_{1,2}^{2}+u_{2,1}^{2}\right)\right. \\
& -r\left(u_{1,1} u_{2,2}-u_{1,2} u_{2,1}\right)-Q\left(\lambda_{2} u_{1,1}+\lambda_{1} u_{2,2}\right. \\
& \left.\left.+u_{1,1} u_{2,2}-u_{1,2} u_{2,1}\right)+f_{\varepsilon}\right] d X_{1} d X_{2} \\
& -\int_{0}^{l_{0}}\left[T_{1}\left(X_{1}\right) u_{1}\left(X_{1}, 0\right)+T_{2}\left(X_{1}\right) u_{2}\left(X_{1}, 0\right)\right] d X_{1},
\end{aligned}
$$

where $l_{0}$ is the period of both the surface displacements and the function $Q$ with respect to coordinate $X_{1} ; T_{i} \quad(i=1,2)$ are forces per unit undeformed area on the substrate surface exerted by the film; $\phi_{s}=\int_{0}^{l_{0}}\left[T_{1}\left(X_{1}\right) u_{1}\left(X_{1}, 0\right)+T_{2}\left(X_{1}\right) u_{2}\left(X_{1}, 0\right)\right] d X_{1}$ is the work done by the surface forces. The Euler equations are obtained from the condition of a vanishing first variation of $\phi$ in Eq. (8), which are given as follows [30]

$$
\left\{\begin{array}{l}
\nabla^{2} u_{1}-\lambda_{2} Q_{, 1}-Q_{, 1} u_{2,2}+Q_{, 2} u_{2,1}=0 \\
\nabla^{2} u_{2}-\lambda_{1} Q_{, 2}-Q_{, 2} u_{1,1}+Q_{, 1} u_{1,2}=0 \\
\lambda_{2} u_{1,1}+\lambda_{1} u_{2,2}+u_{1,1} u_{2,2}-u_{1,2} u_{2,1}=0
\end{array},\right.
$$

where $\nabla^{2}=\frac{\partial^{2}}{\partial X^{2}}+\frac{\partial^{2}}{\partial X_{2}^{2}}$ is the Laplace operator. Based on the following facts: (1) The displacement components are (assumed) periodic in the $X_{1}$ direction with the period/wavelength $l_{0}$; (2) The substrate is modeled as a half-space (the displacements far away from the top surface must be vanished), the boundary conditions are given as

$\left\{\begin{array}{l}u_{i}\left(0, X_{2}\right)=u_{i}\left(l_{0}, X_{2}\right) \\ u_{i}\left(X_{1},-\infty\right)=0\end{array}\right.$,

where $i=1,2$. The expressions for the surface tractions $T_{i}$ are also obtained by the vanishing of the first variation of $\phi$, namely

$\left\{\begin{array}{l}T_{1}=\left.\mu_{s}\left[u_{1,2}+(r+Q) u_{2,1}\right]\right|_{X_{2}=0} \\ T_{2}=\left.\mu_{s}\left[u_{2,2}-(r+Q) u_{1,1}-\lambda_{1} Q\right]\right|_{X_{2}=0}\end{array}\right.$.

The period-doubling morphology occurs due to the coupling of different sinusoidal modes [33]. To analyze the response of the substrate when this non-sinusoidal configuration appears after the wrinkling instability, the surface displacements are assumed to consist of two modes with different wavelength, which gives the following $[30,33]$

$\left\{\begin{array}{l}u_{1}\left(X_{1}, 0\right)=k_{0}^{-1}\left[\delta a_{1} \sin \left(k_{0} X_{1}\right)+\delta^{2} a_{2} \sin \left(n k_{0} X_{1}\right)\right] \\ u_{2}\left(X_{1}, 0\right)=k_{0}^{-1}\left[\delta b_{1} \cos \left(k_{0} X_{1}\right)+\delta^{2}\left(b_{2} \cos \left(n k_{0} X_{1}\right)+c\right)\right]\end{array}\right.$,

where $\delta$ is a small parameter; both $a_{i}$ and $b_{i}$ represent the

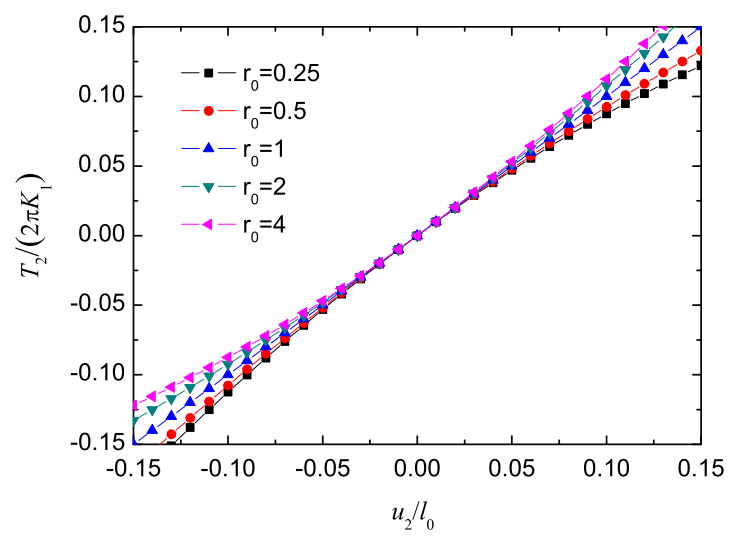

Fig. 2. The effect of pre-stretch ratio $r_{0}$ on the normalized surface tractiondisplacement relationship for a neo-Hookean half-space. dimensionless amplitude factor; $k_{0}=2 \pi / l_{0}$ is the wave number; $n$ is an arbitrary coefficient $(n \neq 1)$ to indicate the difference in wave numbers of these two modes. Particularly, the mode arising from the period-doubling based on the incompressibility condition, which is presented in Appendix. With the application of the perturbation method, the solution of the nonlinear boundary value problem (Eqs. (9)-(12)) is obtained (see Appendix for details). Meanwhile, the surface tractions listed in Eq. (11) can be derived by the substitution of the displacements and the function $Q$. By enforcing the tangential displacement constraint, i.e., $a_{1}=a_{2}=0$, the normal traction $T_{2}$ is given by

$$
\begin{aligned}
T_{2}= & \mu_{s}\left(1+r^{-1}\right)\left(\delta b_{1} \cos \left(k_{0} X_{1}\right)+\delta^{2} b_{2} n \cos \left(n k_{0} X_{1}\right)\right) \\
& +\frac{\delta^{2}}{\lambda_{1}} \frac{3\left(r^{2}-1\right) \mu_{s}}{r\left(3 r^{2}+10 r+3\right)} b_{1}^{2}\left[\cos ^{2}\left(k_{0} X_{1}\right)-1 / 2\right]
\end{aligned}
$$

here we only contains the case of tangential displacement constraint, a full analysis should include the case of tangential traction constraint [30].

To illustrate the nonlinear traction-displacement relation of the neo-Hookean substrate clearly, we consider the case of wrinkling, whose morphology is sinusoidal $\left(b_{2}=0\right)$. The surface transverse displacement becomes $u_{2}=\delta k_{0}^{-1} b_{1} \cos \left(k_{0} X_{1}\right)$, and Eq. (13) can thus be approximately written as

$T_{2} \approx K_{1} k_{0} u_{2}\left(X_{1}, 0\right)+K_{2} k_{0}^{2} u_{2}^{2}\left(X_{1}, 0\right)$,

where $K_{1}=\mu_{s}\left(1+r^{-1}\right)$ and $K_{2}=\left[3\left(r^{2}-1\right) \mu_{s}\right] /\left[\lambda_{1} r\left(3 r^{2}+10 r+3\right)\right]$ are associated with the linearity and nonlinearity. The stretch ratio $r$ is related to the nominal strain by $r=r_{0} /(1-\varepsilon)^{2}$, where $r_{0}=1 /\left(\lambda_{1}^{0}\right)^{2}$ is the pre-stretch ratio. In comparison with the expression obtained by Hutchinson [30], Eq. (14) presents a different traction-displacement relationship, which considers the effect of the nominal strain $\varepsilon$, and it is applicable to the situation when the system is further compressed.

For a substrate in the pre-stretched state $\left(\varepsilon=0\right.$, i.e., $\left.r=r_{0}\right), K_{2}$ is positive for pre-compression case $\left(r_{0}>1\right)$ but becomes negative for pre-tension case $\left(r_{0}<1\right)$. The traction-displacement relationship of Eq. (14) is sensitive to the change in the magnitude of the pre-stretch. We define the positive $u_{2}$ as the outward displacement and the negative $u_{2}$ as the inward one. A nonlinear response occurs due to the pre-stretch, as shown in Fig. 2. Pre-tension generates the softening effect $\left(\left|T_{2}\right|<\left|K_{1} k_{0} u_{2}\right|\right)$ on the outward displacement $\left(u_{2}>0\right)$ and the hardening effect $\left(\left|T_{2}\right|>\left|K_{1} k_{0} u_{2}\right|\right)$ on the inward one $\left(u_{2}<0\right)$, while pre-compression leads to a contrary effect on the same displacements. In addition, a linear response occurs when no pre-stretch exerted on the substrate $\left(r_{0}=1\right)$. These trends also match the finite element results of Zang et al. [24] and the theoretical solutions of Hutchinson [30]. However, these trends can be altered as the external compressive strain $\varepsilon$ increases. Fig. 3 illustrates the traction-displacement relationship in both the pre-tension and the pre-compression cases. Precompression causes same effects on the surface displacements of the substrate as $\varepsilon$ increases. Fig. 3(a) shows that the outward displacement is always stiffened and the inward one is softened, even though $\varepsilon$ is as large as 0.6. But when the strain reaches to a high level (e.g., $\varepsilon=0.6$ ), pre-tension produces contrary effects compared with the case of $\varepsilon=0$, as seen in Fig. 3(b).

\section{Numerical simulations of the post-buckling modes}

To further investigate the formation of different post-buckling modes, the finite element simulation of the system is performed. A two-dimensional plane strain model as well as the boundary conditions is illustrated in Fig. 4. The bilayer is compressed 
a

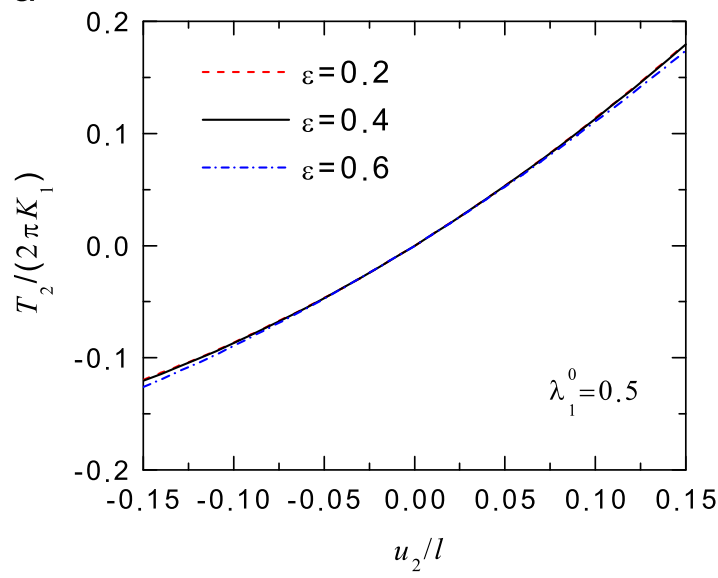

b

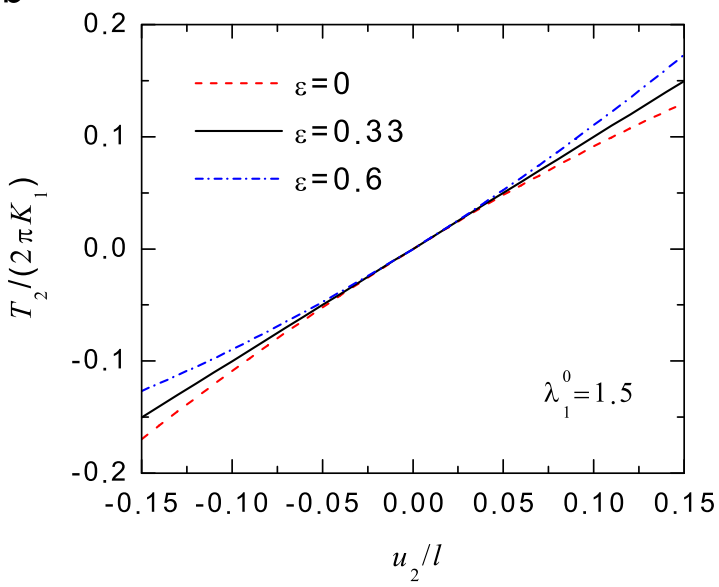

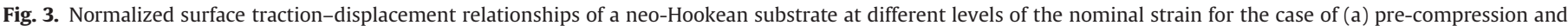
(b) pre-tension.

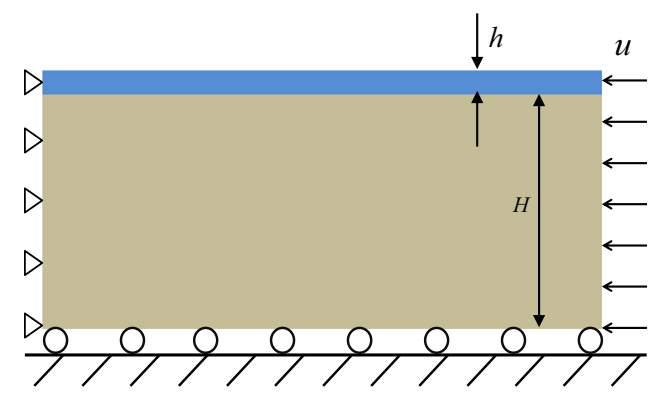

Fig. 4. Schematic of the boundary conditions of a stiff film/soft substrate system for the finite element simulations. Both the shear traction and the horizontal displacement on the left side are zero. On the bottom surface, the shear traction and the vertical displacement are vanished while the top surface of the film is traction free.

by the horizontal displacement load $u$ along the right side, $\mathrm{w}$ hile the left side is constrained in the horizontal direction. Thus the nominal compressive strain is defined as $\varepsilon=u / L$, where $L$ is the original length of the system. The ratio of the substrate depth $H$ to the film thickness $h$ is set to be about 200, so that the substrate is sufficiently deep and can be regarded as a half-space.

The simulations are carried out via the commercial finite element software of ABAQUS. Given that the maximum strain in this study is as large as $\sim 0.3$, both the film and the substrate are modeled as a hyperelastic solid. The incompressible neo-Hookean constitutive model provides a good description of the hyperelastic materials within the range of strain considered here [25,36]. The hybrid plane strain quadrilateral element (CPE8RH) is adopted and the meshes near the film/substrate interface are refined. We use the method reported in [25] to impose the substrate pre-stretch. The post-buckling analysis is then carried out using the arc-length method ("Riks").

In our simulations, the film is perfectly-bonded to the substrate, i.e., no buckle delamination occurs during the compression of the system. Moreover, the film modulus is much higher than that of the substrate, and hence the creasing instability can hardly set in. Consequently, three different post-buckling modes will occur. The sinusoidal wrinkling mode firstly appears and keeps stable at relatively large strain, as shown in Fig. 5(a) for $\varepsilon=0.19$. When $\varepsilon \approx 0.22$, the sinusoidal mode loses stability and grows into the period-doubling mode, as shown in Fig. 5(b). Additionally, Budday et al. [23] revealed that some distinct instability patterns, including period-doubling, period-tripling, and period-quadrupling, can occur as secondary bifurcations by altering the domain width of the simulation model. But period-doubling is energetically favorable over other modes [23]. As the nominal strain increases to approximately 0.28 , several folds are fully developed on the surface of the system (Fig. 5(c)), which is similar to the experiment of Pocivavsek et al. [27].

\section{The onset of period-doubling instability}

Although the critical condition of wrinkling instability has been thoroughly studied (e.g., $[1,17,37,38])$, it is still not clear when the period-doubling instability will occur and how the critical condition varies with the system parameters. In this section, the dimensional analysis is performed to investigate the effects of the system intrinsic parameters, including the film thickness $h$, the substrate pre-stretch $\lambda_{1}^{0}$ and the shear moduli ( $\mu_{f}$ for the film and $\mu_{s}$ for the substrate), on the onset of period-doubling instability. Given that the critical strain of period-doubling instability $\varepsilon_{P D}$ is a dimensionless quantity, but there is no other independent length parameter to normalize the film thickness, i.e., $\varepsilon_{P D}$ is independent of $h$. As a result, $\varepsilon_{P D}$ can be written as the following implicit function

$\varepsilon_{P D}=f\left(\frac{\mu_{f}}{\mu_{s}}, \lambda_{1}^{0}\right)$

which involves two independent variables, the modulus ratio $\mu_{f} / \mu_{s}$ and the pre-stretch $\lambda_{1}^{0}$. However, the function presented in Eq. (15) is difficult to determine analytically. Here, the effects of the parameters $\mu_{f} / \mu_{s}$ and $\lambda_{1}^{0}$ are examined by the finite element simulations as follows.

Fig. 6(a) shows the variations of $\varepsilon_{P D}$ as a function of the modulus ratio $\mu_{f} / \mu_{s}$ for different values of the pre-stretch. It indicates that $\varepsilon_{P D}$ almost keeps constant as the modulus ratio varies. In addition, a recent investigation [23] shows that the critical strain of period-doubling is marginally sensitive to the alternation of the modulus ratio only in the system with the low film/substrate stiffness ratio. Therefore, the effect of the modulus ratio is negligible here for the system with very large ratios of $\mu_{f} / \mu_{s}$, which agrees with the result presented by Cao and Hutchinson [25]. In this situation, $\varepsilon_{P D}$ is the function with the only variable of pre-stretch. Fig. 6(b) plots the variations of $\varepsilon_{P D}$ with respect to $\lambda_{1}^{0}$ for different modulus ratios (e.g. 500 and 1000). By fitting the data presented by the finite element model, the relation 
a

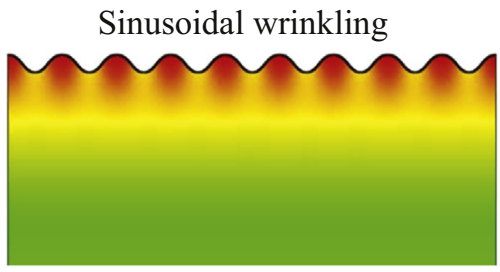

b

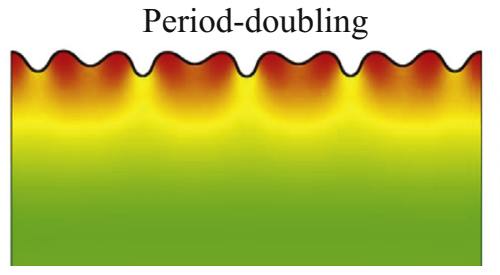

C

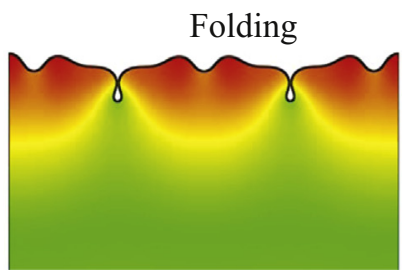

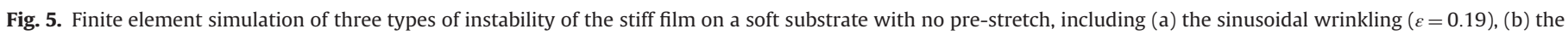
period-doubling $(\varepsilon=0.22)$ and (c) the folding $(\varepsilon=0.28)$. The shear modulus ratio is $\mu_{f} / \mu_{s}=1000$.

a

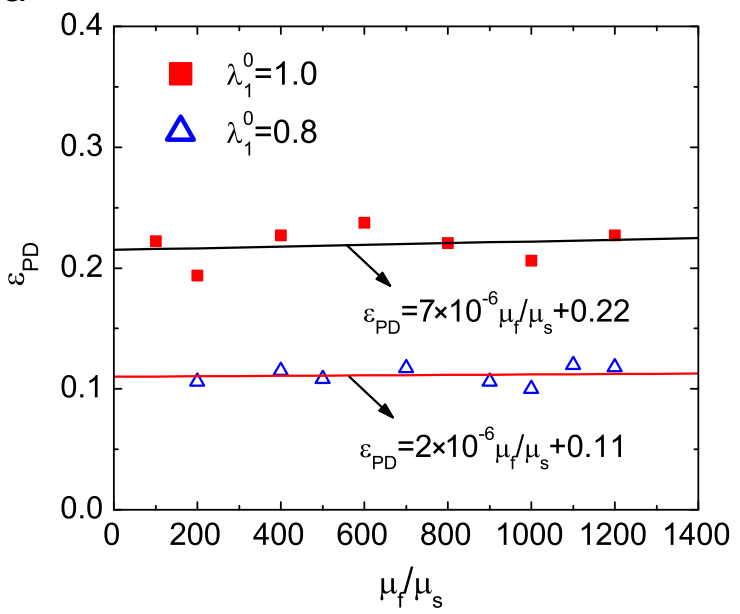

b

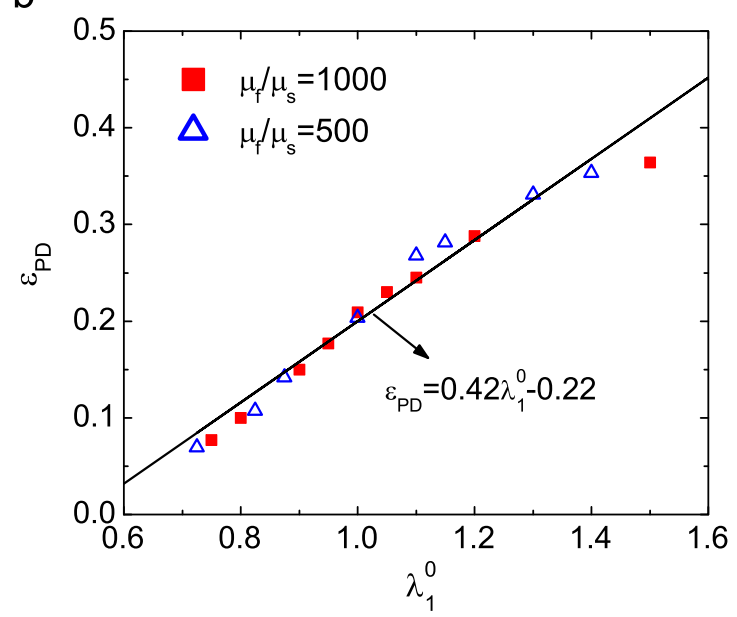

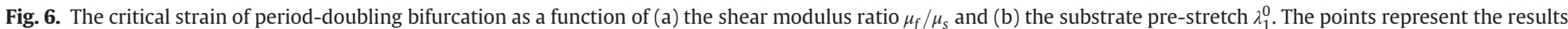
obtained by finite element method and the lines are the corresponding fitting curves.

between $\varepsilon_{P D}$ and the pre-stretch is given as follows:.

$\varepsilon_{P D}=0.42 \lambda_{1}^{0}-0.22$,

Eq. (16) indicates that $\varepsilon_{P D}$ is a linear function of the pre-stretch; moreover, the variation of the modulus ratio makes no difference to the proportion, which further corroborates that the effect of the modulus ratio can be neglected. These trends are in accordance with the experiment and simulation carried by Auguste et al. [36]. If the substrate is not pre-stretched $\left(\lambda_{1}^{0}=1\right)$, it can be seen from Eq. (16) that the critical strain of period-doubling reaches about 0.2, which is in good agreement with the previous experimental and numerical predictions [22,25]. If the substrate is pre-deformed, the critical strain should be smaller for pre-compression and larger for pre-tension. It should be pointed out that those conclusions hold true only under the assumption that the film/substrate modulus ratio is very large.

\section{The transition from period-doubling to folding}

Under further compression, the film/substrate system will evolve into the folding mode beyond the onset of perioddoubling instability. In general, the period-doubling and folding morphologies appear when the system favors inward displacement [24]. It implies that $K_{2}$, which is associated with the substrate nonlinearity, must be positive so as to produce softening for the inward displacement and hardening for the outward one. $K_{2}$ is obtained as presented in Eq. (14), whose variation as a function of the compressive strain $\varepsilon$ is demonstrated in Fig. 7 for several values of the pre-stretch $\lambda_{1}^{0}$. If $\lambda_{1}^{0} \leq 1$ (pre-compression or no pre-stretch), $K_{2}$ remains positive over the full range of nominal strain (from 0 to 0.5 ). The corresponding traction-displacement relation is shown in Fig. 3(a) for $\lambda_{1}^{0}=0.5$. It suggests that the film/

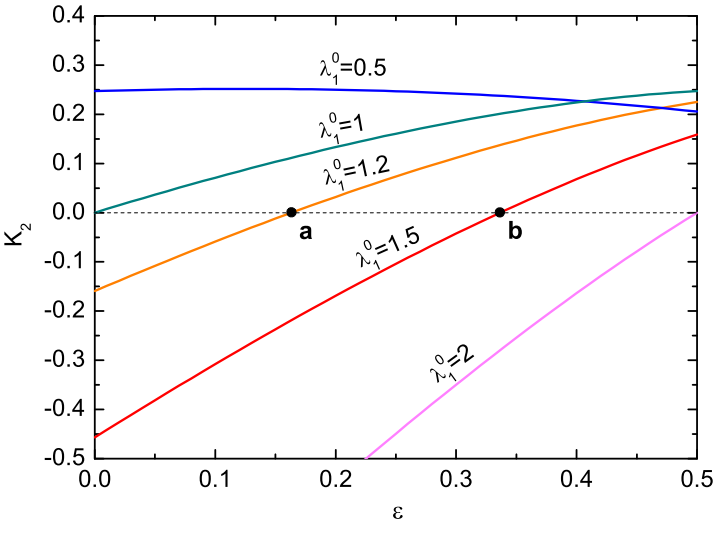

Fig. 7. Variations of $K_{2}$ as a function of the nominal strain for different pre-stretch.

substrate system always favors inward displacements when the substrate is pre-compressed. Hence the folding mode appears under further compression.

However, pre-tension $\left(\lambda_{1}^{0}>1\right)$ leads to the negative $K_{2}$ initially, which can become positive as $\varepsilon$ increases (see Fig. 7). Fig. 3(b) illustrates the surface traction-displacement relations for $\lambda_{1}^{0}=1.5$. It indicates that the softening effect on the inward deformation is generated only when the nominal strain exceeds a threshold. For example, point " $b$ " is such a threshold with $\varepsilon=0.33$ for $\lambda_{1}^{0}=1.5$ (the traction-displacement relationship is linear as shown in Fig. 3 (b)). Moreover, the threshold grows as the pre-tension increases (as point " $a$ " moves to point " $b$ " in Fig. 7). Thus, the critical strain of the period-doubling instability which should be larger than this threshold also increases with increasing pre-tension, as shown in Fig. 6(b). If the nominal strain increases beyond the onset of 


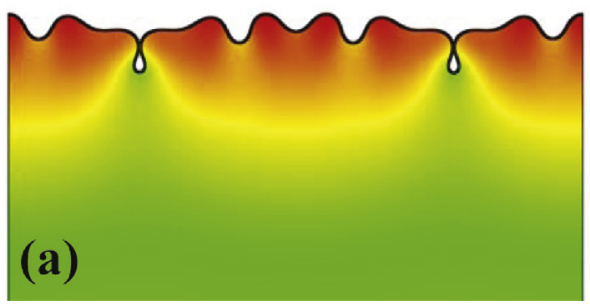

Pre-tension

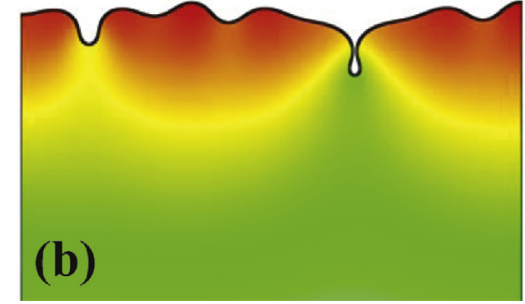

Pre-compression

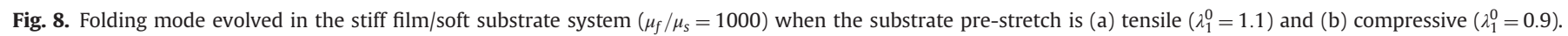

period-doubling mode, the value of $K_{2}$ can be large enough to produce a strong softening for the inward displacement. As a result, the folding mode can also arise after the period-doubling mode. The finite element simulations showed in Fig. 8(a) and (b) further validate that the folding morphology can occur in both the pre-tension and the pre-compression case. Besides, it should be pointed out that $K_{2}$ keeps negative even if the nominal strain reaches a high level (e.g., $\varepsilon=0.5$ ) for a sufficiently large pretension (e.g., $\lambda_{1}^{0}=2$ ), as shown in Fig. 7 . Hence the substrate nonlinearity produces softening for the outward deformation and the system favors the ridge mode rather than folding, which has been investigated numerically in [25].

\section{Conclusions}

The nonlinearity of the neo-Hookean substrate, which depends on both the pre-stretch and the external nominal strain, has significant influences on the formation of the period-doubling morphology and the transition from period-doubling to folding. The positive $K_{2}$ produces softening effect on the surface inward displacement relative to the outward one, and thus facilitates the formation of the period-doubling morphology. For a precompressed substrate, $K_{2}$ remains positive. But for a substrate with pre-tension, $K_{2}$ is initially negative and then becomes positive when the nominal strain exceeds a threshold. Hence, the occurrence of period-doubling instability can occur in both the pre-compression and pre-tension cases, which are also corroborated by the finite element simulations. The positive $K_{2}$ becomes larger as the film/substrate system is further compressed, causing the transition from period-doubling to folding and the folds are fully developed when the compressive strain is sufficiently large. The threshold at which $K_{2}$ changes to be positive grows as the pretension increases, causing the onset of period-doubling instability also rises with increasing pre-tension. In conjunction with the dimensional analysis and the finite element method, we reveal that the critical strain of period-doubling is linearly proportional to the substrate pre-stretch only, or say that the critical strain is independent of the film/substrate modulus ratio and the film thickness. Here an important assumption is used: that the film/ substrate modulus ratio is very large. The exact formula of this critical strain in the general case is still not clear for the neoHookean system and further study is needed.

\section{Acknowledgment}

The research has been supported by the National Natural Science Foundation of China (NSFC no. 11372321).

\section{Appendix}

In view of the form of the surface displacements in Eq. (12), the perturbation form of the solution to Eq. (9) is assumed to be the following:

$$
\left\{\begin{array}{l}
u_{1}(\xi, \eta)=\delta k_{0}^{-1} f(\eta) \sin (\xi)+\delta^{2 \frac{k_{0}^{-1}}{2 \lambda_{1}}}\left[F_{1}(\eta) \sin (n \xi)+F_{2}(\eta) \sin (2 \xi)\right] \\
u_{2}(\xi, \eta)=\delta k_{0}^{-1} g(\eta) \cos (\xi)+\delta^{2} \frac{k_{0}^{-1}}{2 \lambda_{1}}\left[G_{0}(\eta)+G_{1}(\eta) \cos (n \xi)+G_{2}(\eta) \cos (2 \xi)\right] \\
\lambda_{1} Q(\xi, \eta)=\delta h(\eta) \cos (\xi)+\delta^{2} \frac{1}{2 \lambda_{1}}\left[H_{0}(\eta)+H_{1}(\eta) \cos (n \xi)+H_{2}(\eta) \cos (2 \xi)\right]
\end{array}\right.
$$

where $\xi=k_{0} X_{1}, \eta=k_{0} X_{2}$ are the normalized coordinates in the undeformed state. $f, g, h, F_{i}, G_{i}$ and $H_{i}(i=0,1,2)$ are functions of $\eta$ to be determined. Once these functions are determined, the solution to the boundary value problem in the main text is obtained. By substituting Eq. (17) into Eq. (9) and requiring all terms of order $\delta$ to vanish, the ordinary differential equations (ODEs) with respect to $f, g$ and $h$ are listed as follows:

$f^{\prime \prime}-f+r h=0, g^{\prime \prime}-g-h^{\prime}=0, r f+g^{\prime}=0$,

Again, $r$ here is the stretch ratio defined as $r=\lambda_{2} / \lambda_{1}=r_{0} /$ $(1-\varepsilon)^{2}$, where $r_{0}=\lambda_{2}^{0} / \lambda_{1}^{0}$. The boundary conditions in Eq. (10) require $f$ and $g$ to vanish as $\eta \rightarrow-\infty$. Moreover, Eq. (12) requires $f$ and $g$ at $\eta=0$ to satisfy the following conditions:

$f(0)=a_{1}, g(0)=b_{1}$.

Hence $f, g$ and $h$ are solved as follows:

$\left\{\begin{array}{l}f=-A_{1} e^{r \eta}-A_{2} r^{-1} e^{\eta} \\ g=A_{1} e^{r \eta}+A_{2} e^{\eta} \\ h=A_{1}\left(r-r^{-1}\right) e^{r \eta}\end{array}\right.$

where $A_{1}=-\left(r a_{1}+b_{1}\right) /(r-1)$ and $A_{2}=r\left(a_{1}+b_{1}\right) /(r-1)$.

By leaving all terms of order $\delta^{2}$ to vanish, the ODEs governing $F_{i}, G_{i}$ and $H_{i}$ are obtained as

$\left\{\begin{array}{l}G_{0}^{\prime}+g f^{\prime}+f g^{\prime}=0 \\ G^{\prime \prime}{ }_{0}-H_{0}^{\prime}-f h^{\prime}-h f^{\prime}=0^{\prime}\end{array}\right.$
$\left\{\begin{array}{l}F^{\prime \prime}{ }_{1}-n^{2} F_{1}+n r H_{1}=0 \\ G^{\prime \prime}{ }_{1}-n^{2} G_{1}-H_{1}^{\prime}=0, \\ n r F_{1}+G_{1}^{\prime}=0\end{array}\right.$

and

$\left\{\begin{array}{l}F^{\prime \prime}{ }_{2}-4 F_{2}+2 r H_{2}=g h^{\prime}-g^{\prime} h \\ G^{\prime \prime}{ }_{2}-4 G_{2}-H_{2}^{\prime}=f h^{\prime}-f^{\prime} h \\ 2 r F_{2}+G_{2}^{\prime}=f^{\prime} g-f g^{\prime}\end{array}\right.$

$G_{0}$ and $H_{0}$ in Eq. (21) can be further expressed as follows

$G_{0}=-f g, \quad H_{0}=-(f g)^{\prime}-f h$ 
In conjunction with Eqs. (19) and (24), the parameter $c$ in Eq. (12) is obtained as $c=G_{0}(0) /\left(2 \lambda_{1}\right)=-a_{1} b_{1} /\left(2 \lambda_{1}\right)$. Given that Eq. (24) is derived from the third equation of Eq. (9) in the main text, the parameter $c$ is introduced as a consequence of the incompressibility condition. Similar to Eq. (18), Eq. (22) can be solved analytically and the expressions for $F_{1}, G_{1}$ and $H_{1}$ are obtained as follows:

$$
\left\{\begin{array}{l}
F_{1}=-B_{1} e^{n r \eta}-B_{2} r^{-1} e^{n \eta} \\
G_{1}=B_{1} e^{n r \eta}+B_{2} e^{n \eta} \\
H_{1}=B_{1}\left(r-r^{-1}\right) n e^{n r \eta}
\end{array}\right.
$$

The coefficients in Eq. (25) are determined by the boundary conditions, which require the functions to satisfy $\left(F_{1}(0), G_{1}(0)\right)=$ $2 \lambda_{1}\left(a_{2}, b_{2}\right)$. Therefore $B_{1}$ and $B_{2}$ are given as

$B_{1}=-\frac{2 \lambda_{1}}{r-1}\left(r a_{2}+b_{2}\right), B_{2}=\frac{2 \lambda_{1} r}{r-1}\left(a_{2}+b_{2}\right)$.

By substituting the functions $f, g$ and $h$ into Eq. (23), the system of ODEs is reduced to a fourth-order differential equation with respect to one of the functions, say $G_{2}$, namely

$G^{\prime \prime \prime}{ }_{2}-4\left(r^{2}+1\right) G^{\prime \prime} 2+16 r^{2} G_{2}=-\frac{3 c_{1} c_{2}}{r}(r+1)^{2}(r-1)^{3} e^{(r+1) \eta}$.

The solution to Eq. (27) is constructed as the sum of a homogenous solution and a particular solution, satisfying $G_{2}(-\infty)=0$ and $G_{2}(0)=0$, and thus it is given as follows:

$G_{2}=C_{1} e^{2 r \eta}+C_{2} e^{2 \eta}-\frac{3 A_{1} A_{2}(r+1)^{2}(r-1)^{3} e^{(r+1) \eta}}{r\left[(r+1)^{4}-4\left(r^{2}+1\right)(r+1)^{2}+16 r^{2}\right]}$,

where $C_{1}$ and $C_{2}$ are coefficients to be determined later. The functions $F_{2}$ and $H_{2}$ can be determined by substituting function $G_{2}$ into Eq. (23), gives

$F_{2}=-C_{1} e^{2 r \eta}-C_{2} r^{-1} e^{2 \eta}-\frac{A_{1} A_{2}\left(3 r^{3}+5 r^{2}-7 r-1\right)}{r\left(3 r^{2}+10 r+3\right)} e^{(r+1) \eta}$,

and

$H_{2}=2 C_{1}\left(r-r^{-1}\right) e^{2 r \eta}+\frac{A_{1} A_{2}(r-1)^{2}\left(3 r^{2}+6 r+1\right)}{r^{2}(3 r+1)} e^{(r+1) \eta}$.

The coefficients $C_{1}=-\left(A_{1} A_{2}(r-1)\left(3 r^{2}+8 r+3\right) /\left(r\left(3 r^{2}+10 r+\right.\right.\right.$ $3)))$ and $C_{2}=\left(2 A_{1} A_{2}(r-1)\right) /\left(\left(3 r^{2}+10 r+3\right)\right)$, which are determined by the boundary condition, $F_{2}(0)=G_{2}(0)=0$.

\section{References}

[1] X. Chen, J.W. Hutchinson, Herringbone buckling patterns of compressed thin films on compliant substrates, J. Appl. Mech. 71 (2004) 597-603.

[2] J. Genzer, J. Groenewold, Soft matter with hard skin: from skin wrinkles to templating and material characterization, Soft Matter 2 (2006) 310-323.

[3] B. Li, Y.P. Cao, X.Q. Feng, H.J. Gao, Mechanics of morphological instabilities and surface wrinkling in soft materials: a review, Soft Matter 8 (2012) 5728-5745.

[4] E. Cerda, L. Mahadevan, Geometry and physics of wrinkling, Phys. Rev. Lett. 90 (2003) 074302.

[5] J. Yin, Z. Cao, C. Li, I. Sheinman, X. Chen, Stress-driven buckling patterns in spheroidal core/shell structures, Proc. Natl. Acad. Sci. U.S.A. 105 (2008) 19132-19135.

[6] N. Bowden, S. Brittain, A.G. Evans, J.W. Hutchinson, G.M. Whitesides, Spontaneous formation of ordered structures in thin films of metals supported on an elastomeric polymer, Nature 393 (1998) 146-149.

[7] K. Efimenko, M. Rackaitis, E. Manias, A. Vaziri, L. Mahadevan, J. Genzer, Nested self-similar wrinkling patterns in skins, Nat. Mater. 4 (2005) 293-297.

[8] P.-C. Lin, S. Yang, Spontaneous formation of one-dimensional ripples in transit to highly ordered two-dimensional herringbone structures through sequential and unequal biaxial mechanical stretching, Appl. Phys. Lett. 90 (2007) 241903.
[9] C.M. Stafford, C. Harrison, K.L. Beers, A. Karim, E.J. Amis, M.R. Vanlandingham, H.-C. Kim, W. Volksen, R.D. Miller, E.E. Simonyi, A buckling-based metrology for measuring the elastic moduli of polymeric thin films, Nat. Mater. 3 (2004) $545-550$.

[10] J. Huang, M. Juszkiewicz, W.H. de Jeu, E. Cerda, T. Emrick, N. Menon, T.P. Russell, Capillary wrinkling of floating thin polymer films, Science 317 (2007) 650-653.

[11] N. Bowden, W.T.S. Huck, K.E. Paul, G.M. Whitesides, The controlled formation of ordered, sinusoidal structures by plasma oxidation of an elastomeric polymer, Appl. Phys. Lett. 75 (1999) 2557-2559.

[12] T. Ma, H. Liang, G. Chen, B. Poon, H. Jiang, H. Yu, Micro-strain sensing using wrinkled stiff thin films on soft substrates as tunable optical grating, Opt. Express 21 (2013) 11994-12001.

[13] D.-Y. Khang, H. Jiang, Y. Huang, J.A. Rogers, A stretchable form of single-crystal silicon for high-performance electronics on rubber substrates, Science 311 (2006) 208-212.

[14] H. Jiang, D.Y. Khang, J. Song, Y. Sun, Y. Huang, J.A. Rogers, Finite deformation mechanics in buckled thin films on compliant supports, Proc. Natl. Acad. Sci. U.S.A. 104 (2007) 15607-15612.

[15] J. Song, H. Jiang, Z.J. Liu, D.Y. Khang, Y. Huang, J.A. Rogers, C. Lu, C.G. Koh, Buckling of a stiff thin film on a compliant substrate in large deformation, Int. J. Solids Struct. 45 (2008) 3107-3121.

[16] Y. Sun, W.M. Choi, H. Jiang, Y. Huang, J.A. Rogers, Controlled buckling of semiconductor nanoribbons for stretchable electronics, Nat. Nanotechnol. 1 (2006) 201-207.

[17] A.L. Volynskii, S. Bazhenov, O.V. Lebedeva, N.F. Bakeev, Mechanical buckling instability of thin coatings deposited on soft polymer substrates, J. Mater. Sci. 35 (2000) 547-554.

[18] W. Hong, X. Zhao, Z. Suo, Formation of creases on the surfaces of elastomers and gels, Appl. Phys. Lett. 95 (2009) 111901

[19] Y.P. Cao, J.W. Hutchinson, From wrinkles to creases in elastomers: the instability and imperfection-sensitivity of wrinkling, Proc. R. Soc. A-Math. Phys. Eng. Sci. 468 (2012) 94-115.

[20] H.X. Mei, R. Huang, J.Y. Chung, C.M. Stafford, H.H. Yu, Buckling modes of elastic thin films on elastic substrates, Appl. Phys. Lett. 90 (2007) 151902.

[21] H.X. Mei, C.M. Landis, R. Huang, Concomitant wrinkling and buckledelamination of elastic thin films on compliant substrates, Mech. Mater. 43 (2011) 627-642.

[22] F. Brau, H. Vandeparre, A. Sabbah, C. Poulard, A. Boudaoud, P. Damman, Multiple-length-scale elastic instability mimics parametric resonance of nonlinear oscillators, Nat. Phys. 7 (2011) 56-60.

[23] S. Budday, E. Kuhl, J.W. Hutchinson, Period-doubling and period-tripling in growing bilayered systems, Philos. Mag. (2015).

[24] J.F. Zang, X.H. Zhao, Y.P. Cao, J.W. Hutchinson, Localized ridge wrinkling of stiff films on compliant substrates, J. Mech. Phys. Solids 60 (2012) 1265-1279.

[25] Y.P. Cao, J.W. Hutchinson, Wrinkling phenomena in neo-Hookean film/substrate bilayers, J. Appl. Mech. 79 (2012) 031019.

[26] J.-Y. Sun, S. Xia, M.-W. Moon, K.H. Oh, K.-S. Kim, Folding wrinkles of a thin stiff layer on a soft substrate, Proc. R. Soc. A-Math. Phys. Eng. Sci. 468 (2011) 932-953.

[27] L. Pocivavsek, R. Dellsy, A. Kern, S. Johnson, B. Lin, K.Y.C. Lee, E. Cerda, Stress and fold localization in thin elastic membranes, Science 320 (2008) 912-916.

[28] Q. Wang, X. Zhao, Phase diagrams of instabilities in compressed film-substrate systems, J. Appl. Mech. 81 (2013) 051004

[29] Y. Zhang, K.D. Murphy, Secondary buckling and tertiary states of a beam on a non-linear elastic foundation, Int. J. Non Linear Mech. 40 (2005) 795-805.

[30] J.W. Hutchinson, The role of nonlinear substrate elasticity in the wrinkling of thin films, Philos. Trans. R. Soc. A-Math. phys. Eng. Sci. 371 (2013) 20120422.

[31] G.W. Hunt, P.R. Everall, Arnold tongues and mode-jumping in the supercritical post-buckling of an archetypal elastic structure, Proc. R. Soc. A-Math. Phys. Eng. Sci. 455 (1999) 125-140.

[32] P.R. Everall, G.W. Hunt, Mode jumping in the buckling of struts and plates: a comparative study, Int. J. Non Linear Mech. 35 (2000) 1067-1079.

[33] L. Zhuo, Y. Zhang, The mode-coupling of a stiff film/compliant substrate system in the post-buckling range, Int. J. Solids Struct. 53 (2015) 28-37.

[34] W.J. Supple, Initial post-buckling behaviour of a class of elastic structural systems, Int. J. Non Linear Mech. 4 (1969) 23-36.

[35] A.M. van der Heijden, Koiter's Elastic Stability of Solids and Structures, first ed., Cambridge University Press, Cambridge, 2009.

[36] A. Auguste, L. Jin, Z. Suo, R.C. Hayward, The role of substrate pre-stretch in post-wrinkling bifurcations, Soft Matter 10 (2014) 6520-6529.

[37] R. Huang, Kinetic wrinkling of an elastic film on a viscoelastic substrate, J. Mech. Phys. Solids 53 (2005) 63-89.

[38] Z.Y. Huang, W. Hong, Z. Suo, Nonlinear analyses of wrinkles in a film bonded to a compliant substrate, J. Mech. Phys. Solids 53 (2005) 2101-2118. 